\title{
Stand der palynologischen Quartärforschung am schweizerischen Nordalpenrand
}

\author{
(Überblick, Methodisches, Probleme)
}

\section{Vorbemerkung}

Die Entwicklung der Quartärgeologie aus der Sicht eines Menschenlebens ist erstaunlich: Als ich Kind war, publizierten PENCK und BRÜCKNER (1909) das klassische Werk "Die Alpen im Eiszeitalter», wo sie für das Quartär vier Eiszeiten nachwiesen. Doch noch 1938 wurde anläßlich der Frühjahrsversammlung der Schweizerischen Geologischen Gesellschaft in Thun darüber diskutiert, ob es doch nur zwei waren (JAYET), ob es fünf waren (BECK) oder ob es noch mehr waren.

Das Klima der Eiszeiten hatten schon im letzten Jahrhundert Forscher wie NATHORST (1874) und andere aufgrund der "Dryasfloren" als kaltkontinental bezeichnet. Noch 1909 versuchte BROCKMANN-JEROSCH nach Untersuchung der sog. "Diluvialflora" von Kaltbrunn klarzumachen, es sei temperiert-ozeanisch und sehr niederschlagsreich gewesen. BECK (1938b) hat mit der Milankovitch-Kurve ozeanische (die Vereisung fördernde) und kontinentale (erhaltende) Phasen verbunden.

In die Entwicklung der Pollenanalyse in den 1920er Jahren in der Schweiz bin ich hineingewachsen durch meine Lehrer RYTZ und LÜDI. Vor ihnen hatten schon FIRBAS $(1925,1927)$, GaMS und andere das Licht der Pollenanalyse in ältere Ablagerungen $\mathrm{zu}$ tragen versucht. Bei uns hat LÜDI (1953) die bekannten Schieferkohlen der Schweiz untersucht und am Schluß seiner Arbeit (S. 108) resigniert festgestellt, «daß die Pollenanalyse hier als Mittel zur Chronologie des Pleistozäns versagt». Sein Fehlschluß entsprang der Annahme, daß fast alle Schieferkohleschichten Ausdruck von echten interglazialen Warmzeiten seien.

In den dreißig Jahren meiner Tätigkeit am seither neugeschaffenen systematisch-geobotanischen Institut Bern haben sich Tiefbau, Straßenbau und Wasserwirtschaft derart entwickelt, daß allerorten Tiefbohrungen niedergebracht wurden, die die kritisch-stratigraphische Quartärgeologie herausforderten. Hier habe ich eingehängt, mehrmals durch eigene Bohrungen. Für Unterstützung und Interesse sage ich Dank.

\section{Arbeitsweise und Grenzen der Quartärpalynologie}

Pollen und Sporen sind pflanzliche Produkte. Sie besitzen bei aller Kleinheit $(10-150 \mu)$ spezifische morphologische Bau- und Kennmerkmale. In feuchtem Einbettungsmittel sind sie gut erhaltungsfähig. Bei einem großen und wichtigen Teil unserer Vegetationselemente, den Windblütigen, werden sie in so großen Mengen an Luft- und Windströmungen abgegeben, da $\beta$ jedes Quadratzentimeter der Erd- und Wasseroberfläche jährlich viele hundert bis über 10000 dieser Zellen geliefert bekommt.

Die Untersuchung des Pollen-Sporen-Inhalts eines Sediments erlaubt, günstige Archivierung vorausgesetzt, die Pollenablagerungsfolge dieses Akkumulationskomplexes zu ermitteln. Der Untersucher macht uns mit palynostratigraphischen Eigenschaften des Sediments vertraut. Dieses palynostratigraphische Bild eines Akkumulationskomplexes hat zwei Auswertungsmöglichkeiten, eine rein stratigraphische und eine vegetationskundliche.

Die stratigraphische Auswertung ist vorerst beschränkt: sie kann feststellen, daß ein Teil der Ablagerung unter einheitlichen Vegetationsverhältnissen, ein anderer Teil unter andern Verhältnissen sedimentiert wurde.

Die Erkenntnis, daß die Pflanzenarten im Lauf des Quartärs weder durch Evolution noch durch Aussterbeerscheinungen wesentlich verändert worden sind, gibt aber jedem Polleninhalt vielseitigen vegetationskundlichen, ökologischen und klimatologischen Informationsinhalt, der aus dem direkten Vergleich mit der aktuellen Vegetation bezogen werden kann.

Diese vegetationskundliche Auswertbarkeit ergänzt demnach jede nur-lithostratigraphische Betrachtung

em. Prof. Dr. Max Welten

Systematisch-Geobotanisches Institut der Universität

Altenbergrain 21, 3013 Bern

privat: Hohliebestr. 14, 3028 Spiegel-Bern 
mit positiven und negativen Kriterien. Diese werden allerdings oft noch als sekundäre Kriterien gering geschätzt.

Der vegetationskundlich orientierte Pollenanalytiker leitet aus seinen Profilbefunden eine Vegetationsgeschichte quartärer Zeitabschnitte ab. Er muß sich dabei bewußt sein, daß seine Zeitachse das Profil ist, solange er nicht genügend viele Punkte des Profils ${ }^{14} \mathrm{C}$-datieren kann. Dieser absoluten Datierung sind enge Grenzen gesetzt: meist fehlt organisches Material in genügender Menge, noch häufiger überschreitet das Alter des Materials die praktische und methodische Leistungsgrenze von 30000-60000 B.P. des Labors. Die ältere quartäre Vegetationsgeschichte ist deshalb vorläufig eine einfache Aneinanderreihung von Vegetationsbildern analog der lithostratigraphischen Beschreibung des Profilaufbaus. Doch sind Vegetationsbilder viel aussagekräftiger als lithostratigraphische Beschreibungen, weil sie direkter Ausdruck des Allgemeinklimas sind und in viel beschränkterem Maß Resultat lokaler Bedingungen. Die variable lokale Sedimentmächtigkeit formt allerdings (oder verformt) das palynologische Bild des Vegetationsablaufs. Dieser kann aber an andern lokalen Vegetationsabläufen geprüft, ergänzt, im Detail ausgebaut werden.

Die vergleichende Palynostratigraphie kann demnach selbständig eine relative Vegetations-Chronologie des Quartärs aufbauen, die wie die Zähne eines Zahnrades in die Glazial- und Stadialphasen einer anderswie gewonnenen Eiszeitchronologie passen sollte $\left({ }^{18} \mathrm{O} /{ }^{16} \mathrm{O}\right.$-Chronologie, Sedimentations-Chronologie, Aufschotterungs-Chronologie, astronomische Chronologie). Ganz allgemein, besonders aber im gegenwärtigen Zeitpunkt, sind alle Methoden zur Ermittlung der Quartärgeschichte aufeinander angewiesen.

Diesen eingestandenermaßen unsichern Kenntnissen über die geologischen, geomorphologischen, glaziologischen und biologischen Verhältnisse des Quartärs gegenüber legen wir unsern Ausführungen vorläufig das klassische System von PENCK und BRÜCKNER zugrunde. Wir sind aber überzeugt, daß unsere Ergebnisse einer grundlegenden kritischen Betrachtungsweise dienen, wie sie FRENZEL (1980) vertritt.

\section{Neue Ergebnisse zur quartären Vegetationsgeschichte im nordalpinen Vorland der Schweiz}

Ich habe zwar vierzig Jahre über das Holozän und das Spätglazial gearbeitet (wELTEN 1982b), beschränke mich aber hier auf das Pleistozän und dabei auf meine eigenen Untersuchungen. Prof. Dr. S. WEgmüLLER wird über seine Untersuchungen bei Gondiswil und Ufhusen auf der Nordseite des Napfs anläßlich der DEUQUA-Tagung in Zürich selbst berichten. Ich lege eine kurzgefaßte Darstellung meiner Ergebnisse aus drei Hauptuntersuchungsgebieten vor: von Meikirch im Aaregebiet NW von Bern aus dem Zürcher Oberland und obern Glattal aus der Linthebene.

Die wichtigsten Untersuchungslokalitäten werden am letzten Tag der Vorexkursion und am letzten Tag der Nachexkursion der DEUQUA-Tagung 1982 besucht.

\section{Das Mittel- und Jung-Pleistozänprofil von Meikirch bei Bern}

(594.890/205.730/620 m ü. M., $7 \mathrm{~km} \mathrm{NW}$ von Bern)

(Einzelergebnisse in WELTEN 1982a)

Es ist heute am Alpennordrand und in Mitteleuropa das einzige so vollständige und zusammenhängende Profil durch drei Glaziale und zwei Interglaziale. Anlaß zur Untersuchung boten Tiefbohrungen für die Wasserprospektion durch die Geologen P. KELLERHALS und B. TROEHLER. Die Mulde südlich Meikirchs hat sich als Teil einer tiefen, teils vielleicht tektonischen (?), teils glaziofluvialen Rinne am Südostrand des Frienisberges erwiesen. Sie wurde durch den Frienisberg (untere Süßwasser-Molasse), der die Mulde heute $200 \mathrm{~m}$ überragt, vor der Tiefenerosion des von Südwesten vorstoßenden Rhonegletschers geschützt und deshalb vom Eis stets schonend überfahren. Unsere drei untersuchten Profile liegen in Abständen von gegen 100 Metern und weisen recht übereinstimmende Sedimentmächtigkeiten der Einzelabschnitte auf:

\section{Holozän, Postglazial, heutige}

Warmzeit (Boden)

Mächtig- Profil-

keit tiefe

Würm-Eiszeit (Kiese, Sande, wenig Moräne, 20000 B.P.

Moräne oberhalb $9 \mathrm{~m}$ )

Eem, R/W-Warmzeit

(Seeablagerung, Ton-Gyttja)

ca. $1 \mathrm{~m} \quad 0-1 \mathrm{~m}$

Riß-Eiszeit (Seeablagerung,

Sand, Silt, Ton, ohne grobes

Moränenmaterial)

39

Holstein I und II, M/R-Warmzeit (wie Eem)

10

$45-56$

Mindel-Eiszeit-Spätglazial (wie $\mathrm{Ri}$, wenig Kies, ohne eigentliches Moränenmaterial)

$66-112$

Wir erkennen im Profil von oben nach unten (vgl. Diagramm in WELTEN 1981a):

1. Eine mächtige Würmablagerung: im untern Teil glaziofluviale Schotter und Sande (Aare-, Saane-Material?) aus einer ersten intensiven Vereisungsphase, um 30 und $19 \mathrm{~m}$ Tiefe mit tonigen Beruhigungsabschnitten, erst oberhalb $9 \mathrm{~m}$ mit eindeutigem Moränenmaterial, das dem jüngsten Würmvorstoß um 20000 B.P. entsprechen dürfte. 


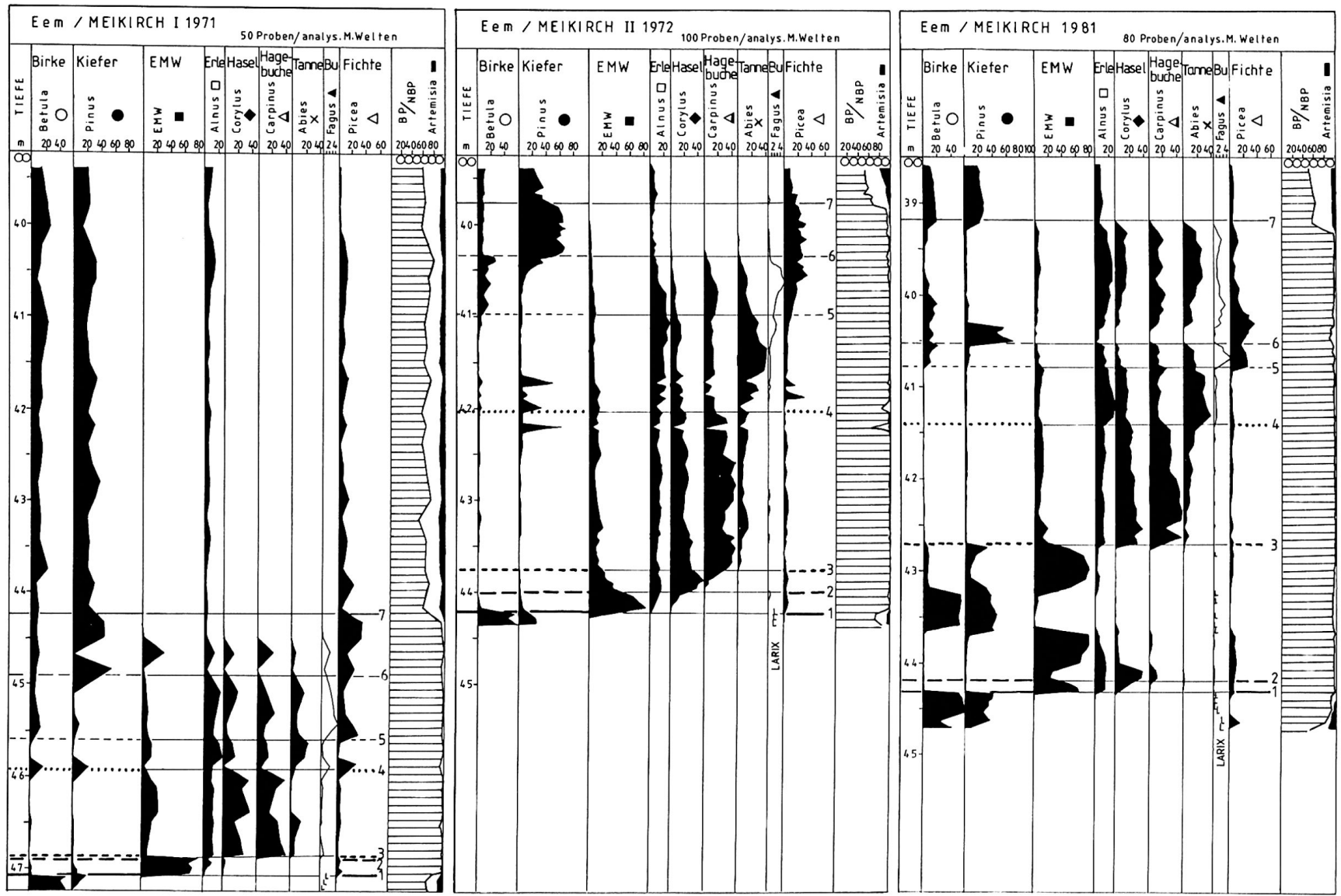

च Abb.1 Meikirch bei Bern. R/W-Interglazial in den 3 untersuchten Tiefbohrungen M I, M II und M 81.-Ziff. 1-7 sedimentologische Störphasen. 
2. Die Eem-Warmzeit (R/W-Interglazial) zeigt die in Mitteleuropa typische Vegetationsfolge: Eichenmischwald/Haseldominanz/Hagebuchenwald/Tannenphase/Fichtenwald mit Buchenspuren/Waldföhrenwald/Birken-Föhren-Tundra.

3. Der rißeiszeitliche Abschnitt, dem jegliche Moränenspuren und wahrscheinlich auch der Abschnitt der eigentlichen Gletscherbedeckung fehlen, zeigt ausgeprägte Interstadialabschnitte: im untern Teil drei Fichten-Interstadiale, nach oben ein langes BirkenLärchen-Interstadial.

4. Der Holstein-Warmzeit Nordeuropas entspricht wohl das "lange Interglazial» der ältern Literatur. Sie ist in Meikirch deutlich zweiteilig: wir bezeichnen die beiden Teile als Holstein I und Holstein II (dem letzten ist wohl die nordeuropäische Treene-, die Waken- oder die Dömnitz-Warmzeit korrelierbar).

5. Die Mindel-Eiszeit ist durch eine mächtige Rinnenfüllung aus dem Mindel-Spätglazial vertreten, die wahrscheinlich bis auf die Molasse-Unterlage hinunterreicht (die Bohrung hat den Fels nicht erreicht).

\section{Das Riß/Würm-Interglazial (= Eem-Warmzeit) in Meikirch bei Bern (Abb. 1)}

Wir stellen in Abb. 1 die pollenanalytischen Ergebnisse an unsern drei Tiefbohrungen von Meikirch (M I, M II, M 81) nebeneinander. Die eigentliche Warmzeit ist durch die Störhorizonte 1-7 gegliedert und korrelierbar.

Unser Diagramm M II (Mitte) zeigt gut die mitteleuropäische Vegetationsfolge der Eem-Warmzeit. Das Eem ist auch im zentralen und niederschlagsärmeren schweizerischen Mittelland eine Warmzeit mit deutlich gestaffelten Vegetationsabschnitten. Diese kennzeichnen sich meist durch das Auftreten und Dominieren einer neuen Baumart. Die Silhouettenkurven sind im Sinn dieser zeitlichen Dominanzfolge angeordnet.

Beim Vergleich unserer drei Diagramme stoßen wir auf ungefähr sieben Profilstörungen, die meist an den Grenzen der Vegetationsabschnitte liegen. Sie bestehen in Hiaten (Profilausfällen, Wachstumsstillständen) und Einschiebungen von Fremdmaterial mit sekundärem Pollengehalt. Besonders mächtige Einschwemmungen zeigt das Profil M 81 zwischen 4400 und $4272 \mathrm{~cm}$ und zwischen 4030 und $3930 \mathrm{~cm}$, die ohne ausführliche Pollenanalysen nicht erkannt worden wären. Auch ist man ganz zu Unrecht versucht, die Kiefernphase zwischen 4050 und $4035 \mathrm{~cm}$ als Kaltphase des jüngern Eem aufzufassen.

Der palynologische Vergleich der drei Meikirch-EemProfile zeigt eindrücklich, welch großen Schwierigkei- ten die Feinuntersuchung pleistozäner Ablagerungen begegnen kann (in allen Untersuchungsmethoden!).

\section{Eem/Frühwürm-Interstadiale im Zürcher Oberland und obern Glattal (Abb. 2)}

Dieses Gebiet stellt in den obern Lagen ein klassisches Schieferkohlengebiet dar (BAUMBERGER, GERBER, JEANNET und WEBER 1923). Das ganze Gebiet wurde durch Trinkwasserbohrungen und Straßenbausondierungen erschlossen und mir durch die Geologen L.WYSSLING, T.LOCHER und C.SCHINDLER zur Verfügung gestellt. Es liegt an der Westabdachung des Zürcher und des Toggenburger Berglandes (obere Süßwassermolasse), anderseits im Überfahrungsgebiet eines Transfluenzarmes des Rhein-Linth-Gletschers. Dieser Gletscherarm überflutete das Gebiet bis weit ins untere Glattal (vgl. die "Geologische Karte des Kantons Zürich und seiner Nachbargebiete» von HANTKE et al. 1967). Dabei erodierte er eine alte Glazialrinne über Grüt-Gossau-Uster-Glattal extrem, z.T. bis auf den Felsgrund, schürfte aber die Alluvialhänge gegen den Bachtel schonend (z.T. vielleicht auch aufschotternd). Die von ihm nicht erodierten und nicht gestauchten Torflager (die heutigen Schieferkohlelager) wurden jeweils von Molasse-Alluvionen eingedeckt. In dieser geologischen Situation konnten wir zwölf Profile ganz oder teilweise pollenanalytisch untersuchen. Sie erstrecken sich über RißEnde, Eem, Früh- und Mittelwürm. Auch hier müssen die Einzelergebnisse in wELTEN 1982a nachgesehen werden (ein Übersichtsdiagramm ist in wELTEN 1981a dargestellt).

Wir erwähnen die Hauptergebnisse von Dürnten, die weitgehend mit der Abb. 2a von Sulzberg-Baden übereinstimmen:

1. In den Schieferkohle-, Ton- und Molasse-Akkumulationsprofilen von Dürnten konnten wir eine heute viel diskutierte Ereignisfolge klar nachweisen: RißSpätglazial/Eem/Frühwürmglazial mit vier Interstadialen/Mittelwürm-Glazial. Das Eem ist gestört, doch eindeutig erkennbar.

2. Die erste Frühwürm-Kaltzeit ist durch starke fluvioglaziale Einflüsse gekennzeichnet und pollenanalytisch gut charakterisiert. Ich benenne sie lokal als Turicum-1-Kaltzeit (T 1).

3. Die in Dürnten nachgewiesenen Fichten-Interstadiale wurden von mir in den Hauptphasen mit Nordeuropa korreliert (wELTEN 1981b): die drei ersten tragen die nordeuropäischen Namen Amersfoort, Brörup und Odderade, das vierte Interstadial scheint in Dürnten zum ersten Mal palynologisch ausführlich dargestellt zu sein und wird von mir Dürnten-Interstadial genannt. 
4. Eine in Groningen mit Anreicherung ausgeführte Radiokarbondatierung knapp über dem Dürnten-Interstadial ergab ein Alter von:

GrN-9865, enriched $55500 \pm 500$ B.P.

Wir gliedern deshalb in voller Übereinstimmung mit nordischen Vorschlägen (MANGERUD, ANDERSEN, BERGLUND und DONNER 1974) die Würmeiszeit in folgende chronostratigraphische Abschnitte:

Frühwürm Mittelwürm Spätwürm

ca. 100000 B.P., 55000 B.P., 25000 B.P., 10000 B.P., evtl. 70000 .

(Ein datiertes Mittelwürm-Diagramm konnten wir bis jetzt nur in Mutten-Signau im mittlern Emmental bearbeiten.)

5. Ein ungewöhnlich schönes, zusammenhängendes und lakustrines Eem/Frühwürm/Mittelwürm-Diagramm konnte ich aus einer 124-m-Tiefbohrung bei Uster (466 m ü. M.) gewinnen, die bis in den Molassefels hinunterreichte. wYSSLING L. u. G. (1978) konnten die Existenz einer fluvioglazialen Rinne durch mehrere Tiefbohrungen nachweisen, die bis unter Kote $300 \mathrm{~m}$ ü. M. auf Molassefels vorstießen. Wir konnten bei Uster nachweisen, daß die erste Rinnenfüllung über Fels aus Riß-Spätglazial-Seetonen besteht, ein Rißgletscher also die Rinne bis auf den Fels ausgeräumt hatte (WELTEN 1982a).

6. Nach zwei 90-m-Tiefbohrungen (bis auf den Fels) bei Nänikon (Großriet) und bei Schwerzenbach (Krutzelried) fehlen aber Glattal-abwärts Riß- und EemAblagerungen gänzlich, so daß der Schluß naheliegt, daß hier der T 1-Frühwürmgletscher bis auf den Fels ausgeräumt hat.

Informationen über Fließdynamik und Erosionsintensität von Gletschern im Randgebiet einerseits, in alten Glazialrinnen anderseits, treten uns aus dem Längsprofil Zürcher Oberland/Glattal entgegen, die Diskussionen und neue Fragen auslösen werden.

Als Anhang zu den Zürcher Ergebnissen zeigen wir noch die Vegetationsentwicklung in der Torfablagerung auf dem Sulzberg (Sulperg) bei Wettingen/Baden, $3 \mathrm{~km}$ außerhalb der maximalen Würmmoränen bei Killwangen (Abb. 2a). Sulzberg und das zum Vergleich daneben reproduzierte Diagramm (Abb. 2b) von Grande Pile (woIllard 1975) aus den westlichen Vogesen illustrieren schön das pulsierende Ausklingen des letzten Interglazials, wie wir es ähnlich auch am Ende des Holstein-Interglazials feststellten (vgl. den nächsten Abschnitt über die Linthebene).

\section{Mindel-Eiszeit/Holstein-Warmzeit am Rand der Linthebene (Abb. 3)}

Nicht weniger klassisch als die besprochenen sind die Schieferkohlen und Pflanzenreste dieses Gebietes (HEer 1858, Heim ALb. 1919, JEANNET in BAUMBerger et al. 1923, BROCKMANN-JEROSCH 1910). Ich konnte 1981 zwei Tiefbohrungen des Wasserwirtschaftsamts St.Gallen und drei eigene Bohrungen bearbeiten. Drei Profile liegen am rechten Talhang zwischen Uznach und Kaltbrunn (auf $468 \mathrm{~m}, 534 \mathrm{~m}$ und $545 \mathrm{~m}$ Meereshöhe; Linthebene $415 \mathrm{~m}$ ), zwei am linken Hang des Obersees ( $480 \mathrm{~m}$ und $489 \mathrm{~m}$ ) in der Gegend Wangen-Buchberg.

Die große Überraschung der Linthebene war für mich die Feststellung, daß an diesen zwei reichlich bearbeiteten Stellen keine Spur von Eem oder Frühwürm nachzuweisen ist, da $\beta$ alle Ablagerungen aus der Mindel-Eiszeit und den zwei Holstein-Warmzeiten stammen. Diese Zuweisung war nur möglich anhand der zwei guten Mindel/Holstein-I/II-Profile von $\mathrm{Mei-}$ kirch. In Abb. 3 stelle ich, etwas vereinfacht, den Abschnitt Mindel/Holstein von Meikirch den Ergebnissen von zwei Profilen der Uznacher-Gegend gegenüber. Die zwei verglichenen Profile liegen $130 \mathrm{~km}$ entfernt, Uznach liegt $100 \mathrm{~m}$ tiefer, Meikirch hat $110 \mathrm{~cm}$ Jahresniederschlag, Uznach $161 \mathrm{~cm}$.

Die kennzeichnenden Merkmale dieser Vergleichsdiagramme sind:

1. Keines der Diagramme kann mit Eem korreliert werden.

2. Beide Diagramme bauen auf langen Spätglazialkomplexen (über Fels!) mit ungewöhnlich stark dominierenden Pinuswerten und sehr geringen Betulawerten und mit hohen Artemisia- und Ephedrawerten auf (Meikirch, Bohrung 1981: 112-64 m, Uznach-Oberkirch 1981: 73-19 m).

3. Beide Warmzeit-Frühphasen sind überraschend ähnlich: nach einer Eichenmischwald-Fichten-Haselphase erreicht sehr früh die Weißtanne (= Tanne, Abies) sehr hohe Werte (Kennzeichen von Holstein I in unsern Gegenden!). Die Tanne wird aber bald in der feuchtern und rauhern Linthebene von Fichte und Grauerle dominiert, so daß sie in manchen Diagrammabschnitten geringe Werte besitzt, doch stets vorhanden ist.

4. Nach stark zunehmender Fichtendominanz erscheint eine Kaltphase mit Fichtenrückgang und ausgesprochener Föhren- und/oder Birkendominanz mit deutlicher Krautpollen- und Artemisia-Zunahme. Eichenmischwaldarten, Hasel und Tanne verschwinden so gut wie ganz (Meikirch um 57,8 m/Mettlen um $13 \mathrm{~m}$ ).

5. Nach dieser (kurzen und mäßigen!) Kaltphase setzt mit anfänglich noch starken Klimarückschlägen eine Warmzeit mit den bisherigen Warmzeitelementen, doch ohne Tanne, ein. Sie war in Uznach abermals stark von Fichte und Erle dominiert, nicht ohne mehrmals Hasel, Eiche und Ulme stärker aufkommen zu lassen.

Diese kürzere Holstein-II-Phase wird nach oben durch sehr hohe Nichtbaumpollenwerte mit ansteigender Artemisia abgeschlossen (Riß). 


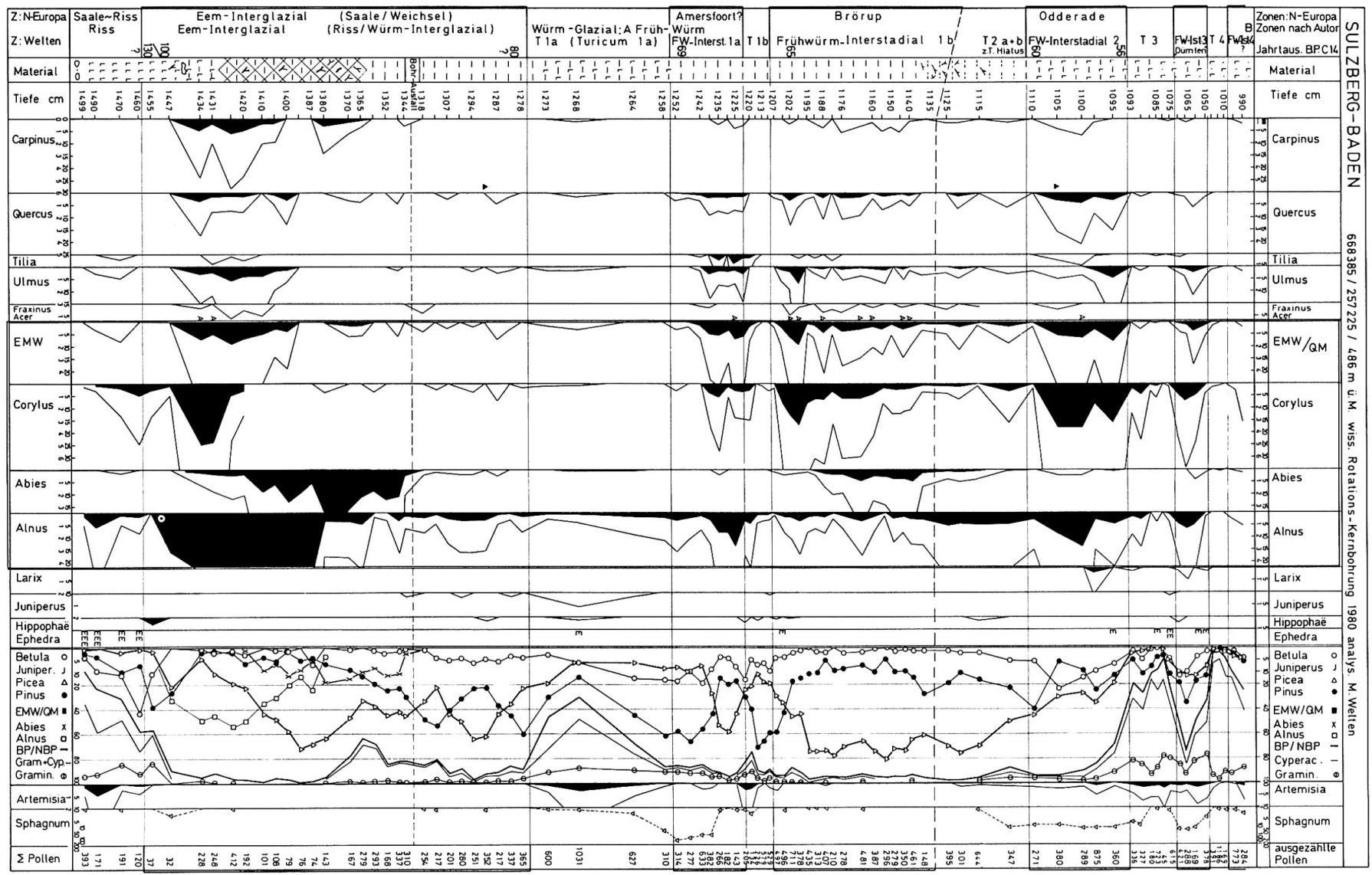

Abb. 2a Sulzberg-Baden: (Eem)/Frühwürm-Interstadiale mit ausklingenden Resten anspruchsvoller Eem-Warmzeitvegetation (WELTEN 1981b) 


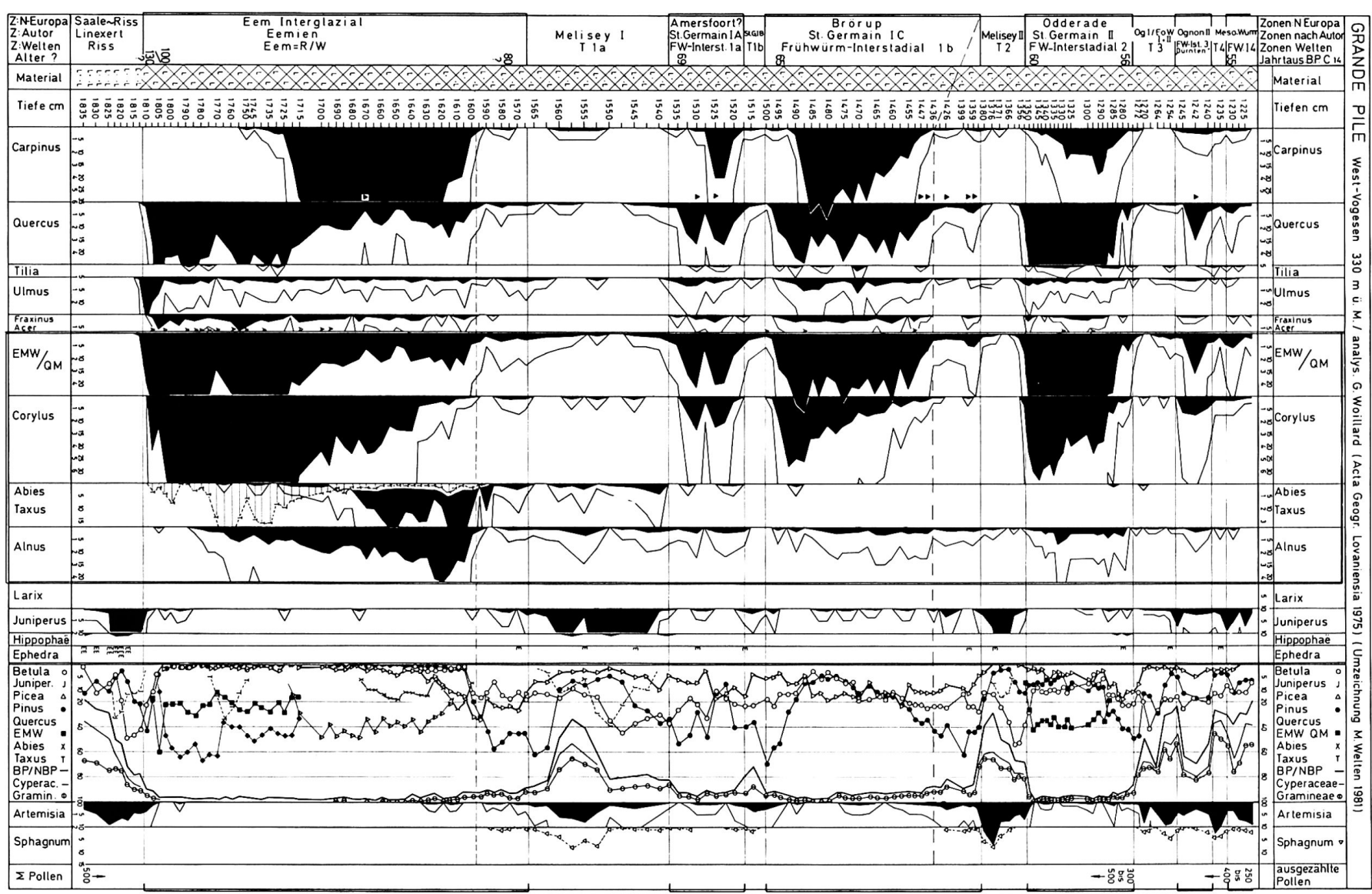

Abb. 2b Grand Pile, Westvogesen: Eem und nachfolgende warmgetönte Frühwürm-Abschnitte (nach WOILLARD 1975, umgezeichnet in WELTEM 1981b) 


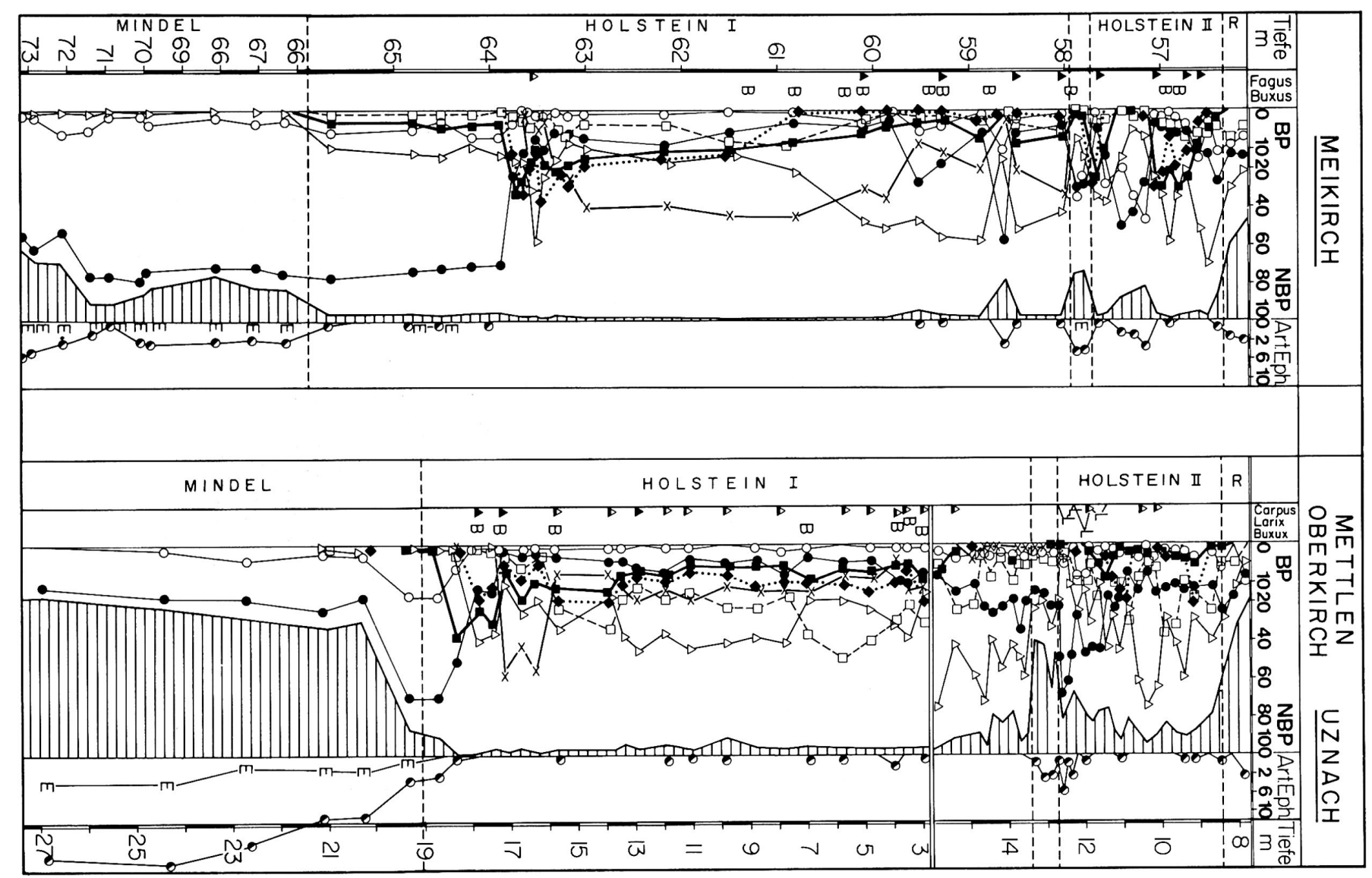

Abb. 3 Meikirch und Linthebene. Übereinstimmung der Vegetationsentwicklung am Ende der Mindel-Eiszeit und in der «Holstein-Warmzeit". 
Wir heben noch folgende Einzelheiten und Eigentümlichkeiten heraus: Die Kaltphase zwischen Holstein I und II weist in Uznach deutliche Lärchenpollenspuren auf, die diese Phase mit den Kaltphasen des Frühwürms im Zürcher Oberland vergleichen lassen. Überhaupt weist unser «Holstein» mehrfache starke Klimaschwankungen auf, deren Intensität im Trennabschnitt Holstein I/II gipfelt und danach wieder abnimmt. Vielleicht gehören die Fichten-Interstadiale des Frühriß in den Komplex von Undulationsphasen der sog. "langen Zwischeneiszeit». BENDA und BRANDES (1974, S. 61) diskutierten diese Schwankungstendenzen und unterschiedlich benannten Warmzeitabschnitte in norddeutschen Ablagerungen.

Fast zahllos sind die großen und kleinen Probleme der Quartärforschung, die vor uns auftauchen. Wir sind überzeugt, daß die Palynologie vieles zu ihrer Lösung beitragen wird.

\section{Literatur}

BAUMBERGER E., GERBER E., JEANNET A. \& WEBER J. (1923): Die diluvialen Schieferkohlen der Schweiz. In: Beitr. Geol. Schweiz, Geotech. Ser. 8, Bern.

BECK P. (1938): Bericht über die außerord. Frühjahrsversammlung der Schweiz. Geol. Ges. in Thun. In: Ecl. Geol. Helv. 31. 173-198.

BENDA L. \& BRANDES H. (1974): Die Kieselgur-Lagerstätten Niedersachsens. In: Geol. Jb., A 21, 3-85, Hannover.

BEUG H.J. (1979): Vegetationsgeschichtlich-pollenanalytische Untersuchungen am Riß/Würm-Interglazial von Eurach am Starnberger See/Obb. In: Geol. Bavarica 80, 91-106, München. BROCKMANN-JEROSCH H. (1910): Die fossilen Pflanzenreste des glazialen Delta bei Kaltbrunn (Uznach, St.Gallen) und deren Bedeutung für die Auffassung des Wesens der Eiszeit. In: Jb. St. Gall. naturw. Ges. 1909, 1-189, St. Gallen.

FIRBAS F. (1927): Beiträge zur Kenntnis der Schieferkohlen des Inntals und der interglazialen Waldgeschichte der Ostalpen. In: Z. Gletscherk. 15, 261-277.

FRENZEL B. (1980): Quelques remarques sur la situation de la stratigraphie du pleistocène dans la partie méridionale d'Allemagne. In: CHALINE J.: Problèmes de stratigraphie quaternaire en France et dans les pays limitrophes, suppl. Bull. AFEQ N.S.1, 1-372, Dijon.

GRUEGER E. (1979): Spätriß, Riß/Würm und Frühwürm am Samerberg in Oberbayern - ein vegetationsgeschichtlicher Beitrag zur Gliederung des Jungpleistozäns. In: Geol. Bavarica 80, 5-64, München.

HANTKE R. (1967): Geologische Karte des Kantons Zürich und seiner Nachbargebiete. In: Viertelj. naturf. Ges. Zürich, 112/2, 91-122, Zürich.

HEER O. (1858): Die Schieferkohlen von Uznach und Dürnten; Zürich.
HEIM ALB. (1919): Geologie der Schweiz. Leipzig, 2 Bde.

LÜDI W. (1953): Die Pflanzenwelt des Eiszeitalters im nördlichen Vorland der Schweizer Alpen. In: Veröff. Geobot. Inst. Rübel, Zürich, 27, 1-208, Bern.

MANGERUD J., ANDERSEN S. T., BERGLUND B.E. \& DONNER J.J. (1974): Quaternary stratigraphy of Norden, a proposal for terminology and classification. Boreas 3, 109-128, Bergen.

NATHORST A. (1874): Sur la distribution de la végétation arctique en Europe au nord des Alpes pendant la période glaciaire. In: Arch. Sc. Phys. et Nat. Genève, $2^{\mathrm{e}}$ pér. 51, 52-64.

PENCK A. \& BRÜCKNER E. (1909): Die Alpen im Eiszeitalter, Leipzig.

WELTEN M. (1981a): Gletscher und Vegetation im Lauf der letzten hunderttausend Jahre (vorläufige Mitteilung). In: Gletscher und Klima. Jb. Schweiz. Naturf. Ges., 1978, Bern.

WELTEN M. (1981b): Verdrängung und Vernichtung der anspruchsvollen Gehölze am Beginn der letzten Eiszeit und die Korrelation der Frühwürm-Interstadiale in Mittel- und Nordeuropa. In: Eiszeitalter und Gegenwart, 31, 187-202, Hannover.

WELTEN M. (1982a): Pollenanalytische Untersuchungen im Jüngern Quartär des nördlichen Alpen-Vorlandes der Schweiz. In: Beitr. z. Geol. Karte der Schweiz, N. F. 156, Basel.

WELTEN M. (1982b): Vegetationsgeschichtliche Untersuchungen in den westlichen Schweizer Alpen: Bern-Wallis. In: Denkschr. Schweiz. Naturf. Ges., 95, Basel.

WOILLARD G. (1975): Recherches palynologiques sur le Pleistocène dans l'Est de la Belgique et dans les Vosges Lorraines. In: Acta Geogr. Lovaniensia 14, 118 p., Louvainla Neuve.

WYSSLING L. U. G. (1978): Interglaziale See-Ablagerungen in einer Bohrung bei Uster (Kanton Zürich). In: Eclogae geol. Helv. 71/2, 357-375. 